\title{
Analysis of repeatomes in Cannabaceae family
}

\author{
Julia Bocharkina \\ Laboratory of Applied Genomics and \\ Crop Breeding \\ All-Russia Research Institute of \\ Agricultural Biotechnology \\ Moscow, Russia \\ Life sciences \\ Skolkovo Institute of science and \\ technology \\ Moscow, Russia \\ julia.bocharkina@skoltech.ru
}

\author{
Olga Razumova \\ Laboratory of Applied Genomics and \\ Crop Breeding \\ All-Russia Research Institute of \\ Agricultural Biotechnology \\ Moscow, Russia \\ razumovao@gmail.com
}

\author{
Gennady Karlov \\ Laboratory of Applied Genomics and \\ Crop Breeding \\ All-Russia Research Institute of \\ Agricultural Biotechnology \\ Moscow, Russia \\ karlovg@gmail.com
}

\begin{abstract}
Analysis of repeatomes in the Cannabaceae family was done. There were found family-specific, speciesspecific and sex-specific clusters. Primers were created and checked.
\end{abstract}

Keywords - NGS, repeatome, plants, Cannabaceae, sex

\section{Introduction}

Plant genomes are characterized by large amount of repetitive DNA and this amount is known as repeatomes of species [1]. There are 3 major types of repetitive DNA: transposable elements (TE), tandem repeats (TR), ribosomal DNA. Transposable elements have a relatively high duplication rate [2]. These copies can impact on transcription, expression of the genes, also they can disrupt genes[3, 4, 5]. Thus, repeats can be very heterogeneous, in addition, they can be unique, low- or high-copy. They may differ in the length of the motifs in the genome [6]. Many families of repeats DNA are not yet known. Also, the role of these sites in the genome is not fully understood. They are known to play an important role in stabilization and maintenance chromosome structures, they are also involved in chromosome recognition and their correct division during mitosis and meiosis. [7]. It is shown that species differentiation in plants often associated with rapid changes in DNA-repeats [2]. Our aim in this research was to study of repeatomes from Cannabaceae family.

The family Cannabaceae contains at least 3 agriculturalsignificant species: Cannabis sativa L., Humulus lupulus L., Humulus japonicus L.. Hemp's (Cannabis sativa $\mathrm{L}$.) has $2 \mathrm{n}=$ 20 and female haploid genome size is $818 \mathrm{Mb}$, but male haploid genome size is $843 \mathrm{Mb}$ [9]; these facts show us that Cannabis sativa L. has heterogenous $\mathrm{X}$ and $\mathrm{Y}$ chromosomes in such a way that $\mathrm{Y}$ chromosome is bigger than $\mathrm{X}$. Cannabis sativa L. has male-determining Y chromosome [9,10,11].

There is an interesting situation with hops (Humulus). Common hop (Humulus lupulus L.) contains $2 \mathrm{n}=20$ with the $\mathrm{XY}$ system, but sex-determination occurs according to the $\mathrm{A}: \mathrm{X}$ ratio [8]. Humulus japonicus $\mathrm{L}$. contains $\mathrm{XY}_{1} \mathrm{Y}_{2}$ and the female plant has $2 n=16$, the male plant has $2 n=17$ [12]. Why sex determination is so different within one family?

Therefore, one of the important tasks of modern biology is to study repeatomes from the sex-evolution, structural bioinformatics and cytogenetics points of view.

\section{Materials and methods}

For the research, there were used Humulus lupulus plant samples from Moscow region, $H$. japonicus plants cultivar "Samurai" Gavrish, Cannabis sativa L. plant ("Zenitsa" cultivar) DNA were purified from leaves using the Doyle \& Doyle method with modifications [13]. DNA libraries were prepared according to the instructions of commercial kits. For all plants, there were done one full cycle of Illumina MiSeq sequencing.

Sequencing reads were analyzed by quality control tool FastQC

(http://www.bioinformatics.babraham.ac.uk/projects/fastqc/). Quality filtering of the resulting raw fastq reads, adaptors trimming, filtering out short or unpaired sequences and trimming reads to lengths using the Trimmomatic tool. [14]. Then, the two-resulting pair-term sequencing files were combined using the FASTQ interlacer tool, which allows you to automatically filter paired and unpaired sequences. A file with paired reads was used for further analysis.

Bioinformatic analysis of the repeatomes was carried out on a local server with 28 nuclear processors and $128 \mathrm{~GB}$ of RAM, running the Linux operating system using RepeatExplorer [15]. Clusters containing at least $0.01 \%$ of all clustered reads were characterized using RepeatMasker [16] using the Repbase database [17]. The determination of the unit of tandem repeat monomer is performed by RepeatExplorer.

Pairwise scatterplots were built in R using the ggplot2 package using custom scripts [18].

PCRs were done, conditions were optimized: annealing temperature $60^{\circ}, 35$ cycles.

\section{Results}

Identification of common and species-specific repeats were done with usage strategy of comparative clusterization according to the following scheme: a set of Humulus lupulus L. sequences (female + male plant) and a set of Humulus japonicus L. sequences (female + male plant).

The majority of species-specific sequences for both genomes are non-classified repeats-families. And a large amount of known species-specific repeats related to the Ty3/Gypsy family( 8\%).

Finally, 88 species-specific clusters for the common hop and 10 species-specific clusters for Japanese hop were identified. The highest-copy clusters were selected as candidates for the creating of species-specific markers.

There were not completely sex-specific clusters. This may be due to several factors, for example, the number of reads used for analysis. We compared two different species, one can notice a stronger divergence of the repeating sequences for Humulus japonicus L., which may be evidence of an earlier 
discrepancy between the ancestors of Humulus japonicus L. compared to Humulus lupulus L. As a criterion for the selection of sex-specific clusters, we were guided by two parameters: the copy number of the cluster and the difference in the number of sequences of the male and female genomes in this cluster. So, for Humulus japonicus L., 8 of the most replicated clusters were selected with a minimum value of the difference in the number of reads between the male and female genomes. For Humulus lupulus L. there were chosen 4 clusters for further work.

Primers were created and, finally, we had 17 pairs of primers. We decided to check our primers with doing PCR and electrophoresis It was shown that most of the primers are not species-specific.

PCR was done and it was shown that we have 3 categories of primers:

1) Universal for Cannabaceae family (for both hops and hemp);

2) Universal for Humulus lupulus and Humulus japonicus;

3) Species-specific for Humulus lupulus L. or Humulus japonicus L.

This research is in progress.

\section{ACKNOWLEDGMENT}

The work is supported by RFBR No. 20-316-70018\19.

\section{REFERENCES}

[1] Maumus F., Quesneville H. Deep investigation of Arabidopsis thaliana junk DNA reveals a continuum between repetitive elements and genomic dark matter // PLoS One. - 2014. - T. 9. - №. 4.

[2] Lisch D. How important are transposons for plant evolution? // Nature Reviews Genetics. - 2013. - T. 14. - №. 1. - C. 49-61.
[3] Feschotte C. Transposable elements and the evolution of regulatory networks // Nature Reviews Genetics. - 2008. - T. 9. - №. 5. - C. 397 405.

[4] Faulkner G. J. et al. The regulated retrotransposon transcriptome of mammalian cells // Nature genetics. - 2009. - T. 41. - №. 5. - C. 563.

[5] Fedoroff N. V. Transposable elements, epigenetics, and genome evolution // Science. - 2012. - T. 338. - №. 6108. - C. 758-767.

[6] Wicker T. et al. A unified classification system for eukaryotic transposable elements // Nature Reviews Genetics. - 2007. - T. 8. - №. 12. - C. 973-982

[7] Neumann P. et al. Plant centromeric retrotransposons: a structural and cytogenetic perspective // Mobile DNA. - 2011. - T. 2. - №. 1. - C. 4.

[8] Winge O. The nature of sex chromosomes // Proc Sixth Int Congr Genet. - 1932. - T. 1. - C. 343-355.

[9] Sakamoto K. et al. Characterization; genome sizes and morphology of sex chromosomes in hemp (Cannabis sativa L.) // Cytologia. - 1998. T. 63. - №. 4. - C. 459-464.

[10] Ming R., Bendahmane A., Renner S. S. Sex chromosomes in land plants // Annual review of plant biology. - 2011. - T. 62. - C. 485-514.

[11] Divashuk M. G. et al. Molecular cytogenetic characterization of the dioecious Cannabis sativa with an XY chromosome sex determination system // PloS one. - 2014. - T. 9. - №. 1.

[12] Aleksandrov O. S., Divashuk M. G., Karlov G. I. Development of a sex-specific molecular marker for Japanese hop Humulus japonicus Siebold \& Zucc // Russian Journal of Genetics. - 2011. - T. 47. - №. 8. - C. 1016.

[13] Doyle J. J. Isolation of plant DNA from fresh tissue // Focus. - 1990. T. 12. - C. 13-15.

[14] Bolger A. M., Lohse M., Usadel B. Trimmomatic: a flexible trimmer for Illumina sequence data // Bioinformatics. - 2014. - T. 30. - №. 15. - C. 2114-2120.

[15] Novák P. et al. RepeatExplorer: a Galaxy-based web server for genome-wide characterization of eukaryotic repetitive elements from next-generation sequence reads // Bioinformatics. - 2013. - T. 29. - №. 6. - C. 792-793.

[16] Smit A. F. A., Hubley R., Green P. RepeatMasker Open-4.0. 20132015. -2015 .

[17] Jurka J. et al. Repbase Update, a database of eukaryotic repetitive elements // Cytogenetic and genome research. - 2005. - T. 110. - №. 1-4. - C. 462-467.

[18] Wickham H. ggplot2: elegant graphics for data analysis. - Springer, 2016. 